\title{
Constant Light Disrupts the Developing Mouse Biological Clock
}

\author{
HIDENOBU OHTA, AMANDA C. MITCHELL, AND DOUGLAS G. MCMAHON \\ Department of Biological Sciences [H.O., A.M., D.G.M.], Vanderbilt University, Nashville, TN 37235; Department of Obstetrics [H.O.], \\ Tohoku University Hospital, Sendai 980-8574, Japan
}

\begin{abstract}
The central biological clock of the brain, contained within the suprachiasmatic nuclei (SCN) of mammals, orchestrates an orderly "internal day" of physiology and behavior. The developing biological clock begins to respond to light at an early stage and a particular concern in humans is whether light exposure has disruptive effects on the developing biological clock of infants exposed to constant lighting conditions in neonatal intensive care units (NICUs). Worldwide, eighteen million, or $14 \%$, of newborns estimated to be of low birth weight, are exposed to artificial lighting environments in hospital nurseries annually. Here, we have tested whether constant light (LL) exposure disrupts the developing biological clock of mice, using a circadian reporter transgenic mouse model in which the organization of the central biological clock can be assayed by real-time gene expression imaging. We now find that LL has both acute and long-term disruptive effects on developing biological clocks and that cyclic lighting conditions are critical for developing circadian clocks to coordinate their molecular circadian mechanisms. This suggests that, from the perspective of developing circadian organization in humans, cyclic light conditions in NICUs are likely to be most appropriate for infants. (Pediatr Res 60: 304-308, 2006)
\end{abstract}

$\mathrm{T}$ The central biological clock of the brain, contained within the suprachiasmatic nuclei ( $\mathrm{SCN}$ ) of mammals, orchestrates an orderly "internal day" of physiology and behavior through its extensive influence on multiple organ systems, including the brain, heart, liver and lung (1). In many organisms, including humans, the biological clock and its control of circadian organization can be disrupted by exposure to constant light (LL) environments (2-6). Although previous studies have focused on effects of LL on adult organisms, in humans, a particular concern is whether LL has acute or long-term disruptive effects on the developing biological clock of preterm infants, who may be exposed to LL conditions in neonatal intensive care units (NICUs).

Clinical studies have suggested effects of light on preterm infants including disturbances of biologic rhythms and sleep states $(7,8)$, effects on postnatal weight gain $(7,9,10)$, and on visual development $(11,12)$, indicating that the developing biological clock begins to respond to light at an early stage. However, there is no clear-cut scientific consensus on what constitutes an "appropriate" lighting environment for these infants and many NICUs are brightly and/or continuously lit.

Received December 7, 2005; accepted June 5, 2006.

Correspondence: Douglas G. McMahon, Ph.D., Department of Biological Sciences, Vanderbilt University, VU Station B, Box 35-1634, Nashville, TN 37235-1634; e-mail: douglas.g.mcmahon@vanderbilt.edu

Supported by NIH R01 MH63341 to D.G.M
Although there is a trend of late to use lower levels of illumination and to introduce cyclic lighting conditions, current recommendations for illumination in NICUs state that "Appropriate general lighting levels for NICUs have not been established." (11,13).

In this study we have tested whether exposure to LL disrupts the developing biological clock of mice, using a circadian reporter transgenic mouse model in which the cellular and molecular organization of the central biological clock can be assayed by real-time gene expression imaging and correlated with individual animal behavior (14-17). Our findings indicate that exposure of developing mice to LL acutely disrupts circadian behaviors as well as cellular synchrony within the central biological clock and has lasting effects on circadian organization that result in increased vulnerability to disruption by LL. Coherent circadian organization can be reestablished by switching to cyclic light conditions. These studies suggest that, from the perspective of developing circadian organization in humans, cyclic light conditions are likely to be most appropriate for preterm infants.

\section{METHODS}

Transgenic mice. Vanderbilt University bred $\mathrm{B} 6 \mathrm{C} 3 \mathrm{H}$ mice homozygous for the mPeriod1 (Per1):d2EGFP transgene (17) were cross-bred to produce mice homozygous for the reporter transgene (Perl:GFP mice). Pregnant homozygous Per1:GFP mice $(n=10)$ were maintained under 12-h light/12-h dark cycles (LD, lights on at 06:00h). The day of delivery was designated as postnatal day 0 (P0). Each dam fed 5 pups from P0 through P20, the day of weaning. On P0 half of the group ( 5 dams with 25 pups) remained in the same LD conditions while the other half was transferred into constant light conditions (LL, 350 lux). On P20 the Per1:GFP signals in the SCN of pups from each condition were measured in in vitro sliced tissue culture. Animal care and use was reviewed and approved by the Vanderbilt University IACUC.

SCN sliced tissue culture. Mouse pups were humanely killed by cervical translocation and decapitation between 17:00 and 18:00 h (local time). Their brains were rapidly removed and placed in cold HBSS (14060-057; Invitrogen, Grand Island). Coronal sections (200- $\mu$ m thick) including both left and right nuclei were made with a vibratome. SCN were cultured using the same methods as Ohta et al. (17).

Per1:GFP imaging. Cultures were maintained at $36^{\circ} \mathrm{C}$ in darkness, and their GFP signals were monitored for $3.5 \mathrm{~d}$ with a laser-scanning confocal microscope (Carl Zeiss, Jena, Germany). Three optical Z-plane sections of 54 $\mu \mathrm{m}$ that covered a total depth of $94 \mu \mathrm{m}$ were acquired at hourly intervals. For analysis, the three image planes were combined and SCN or individual cells within SCN were delimited by a region of interest tool, and their fluorescence

Abbreviations: E, embryonic day; LD, 12-hr light/12-hr dark cycles; LL, constant light; P, postnatal day; Perl, Period 1; SCN, the suprachiasmatic nuclei

DOI: 10.1203/01.pdr.0000233114.18403.66 
intensity was tracked over time with IP Lab software (Scanalytics, Fairfax, VA). Background fluorescence, assayed at each time-point from regions outside the borders of the SCN, was subtracted from SCN and cell values. Results are expressed as mean pixel intensity in arbitrary units (AU) within SCN and cells to normalize for SCN and cell size differences and plotted using a moving five-point average smoothing function. Cells were classified as non-rhythmic by the criteria previously used in Ohta et al. (17).

Analysis of single cell rhythms. The peak time distributions and standard deviations of Per1:GFP rhythms from single SCN neurons for LD and LL pups were calculated on the circadian cycle that occurred during hours 12-36 in vitro (Fig. $1 D, H$ ). Statistical comparisons for these data from the two experimental groups were performed by unpaired Student's $t$-tests $(p<0.05)$.

Activity rhythms. Wheel-running activity was recorded and analyzed using ClockLab software (Actimetrics, Chicago, IL). $\chi^{2}$ periodogram analysis (18) of 14-d segments of wheel running activity was used to determine behavioral rhythmicity. Animals were judged to lack circadian behavioral organization when their Qp value for a 14-d segment of activity was below $p=0.05$ or when they exhibited multiple period peaks that exceeded the $p=0.05$ value
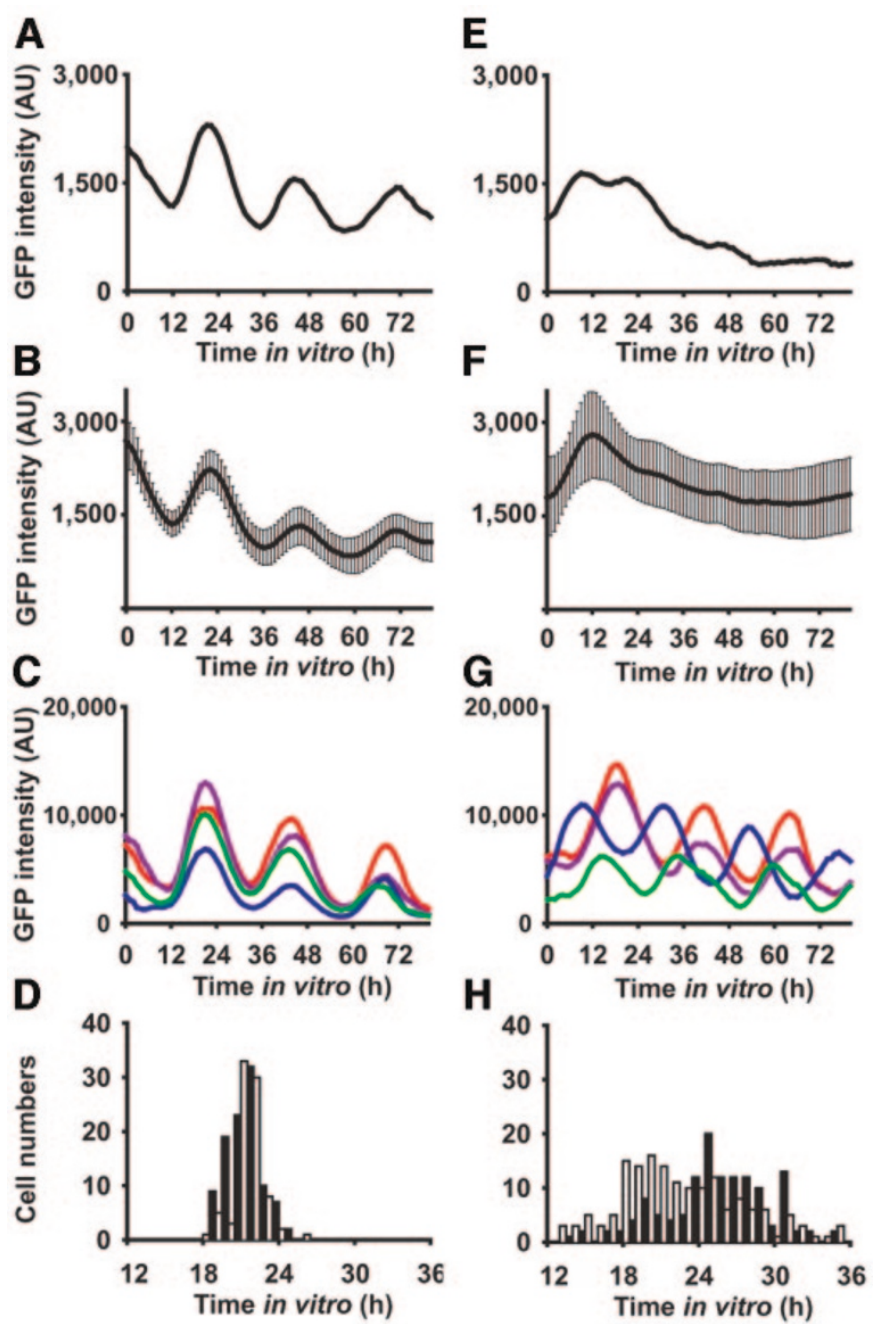

Figure 1. SCN Rhythms from an LD-Treated $(A-D)$ and Constant LightTreated $(E-H)$ Mouse Pups. (A) Time-lapse SCN Perl:GFP fluorescence signals for $3.5 \mathrm{~d}$ in vitro from a mouse pup raised in $\mathrm{LD}$. (B) Group data of Perl:GFP rhythms from 5 LD mouse SCN. (C) Individual SCN neuronal Perl:GFP rhythms from SCN in (A). Four representative cells (colored lines) are plotted for clarity. $(D)$ Peak time histograms of individual neuronal rhythms in $(A)$. Peak times of neurons in right SCN are plotted in black bars whereas those in left SCN are plotted in open bars. Histograms for this and the following figures are for hours $12-36$ in vitro $(n=191$ cells). (E) Time-lapse SCN Perl:GFP fluorescence signals from a mouse pup raised in LL. $(F)$ Group data of Perl:GFP rhythms from 5 LL mouse SCN. $(G)$ individual SCN neuronal Perl:GFP rhythms from SCN in $(E)$. $(H)$ Peak time histograms of individual neuronal rhythms from $\mathrm{SCN}$ in $(E) n=307$ cells. but were not circadian in period (i.e., split rhythms). On P20, five pups were separated from each dam and transferred from their original cage into individual cages for their behavioral recordings. Two groups were prepared to evaluate the effects of the lighting conditions during the first three weeks of age on the development of their behavioral rhythmicity after weaning: in the first group, on P20 LL, pups were transferred into LD conditions (LL-LD pups); in the second group, after P20 LL, pups remained in LL (LL-LL pups). The same type of behavioral observation with mice was previously reported in (19).

\section{RESULTS}

Effects of constant light on the developing biological clock. To test the effect of constant light on the SCN during early development, we used Perl:GFP transgenic mice in which rhythmic activation of the Perl clock gene promoter can be monitored at the single cell level, in real-time, using time-lapse confocal microscopy $(16,17)$. Pregnant dams were maintained on LD 12:12 light cycles during gestation and then, following parturition, dams and pups were exposed to either normal light-dark (LD) cycles or LL for the first three weeks of postnatal life while the locomotor activity rhythms of dams were assayed by wheel running. On P20 the SCN of LD and LL pups were dissected, explanted into organotypic culture and their Perl promoter-driven GFP fluorescence rhythms were recorded during the following $3.5 \mathrm{~d}$ in vitro. Individual SCN from LD pups exhibited clear circadian rhythms in gene expression averaging 3.04-fold in amplitude on the first circadian cycle in vitro $( \pm 0.66$ mean \pm SEM, throughout; 5 mice, Fig. 1A), similar in amplitude to SCN rhythms from LD-entrained adult mice (17). In addition, ongoing circadian rhythmicity was evident in the average SCN fluorescence data from all 5 preparations (Fig. 1B). We also tracked the molecular circadian rhythms of individual neurons within the SCN of LD pups and found that they also exhibited robust Perl:GFP rhythms and a high degree of synchrony (Fig. 1C). Peak time histograms of individual cell rhythms from LD pup SCN showed the majority of cell rhythms were synchronized in a single peak with a mean SD of $1.9 \pm 0.1 \mathrm{~h}$ (Fig. 1D). Similar results were observed for individual cells in SCN of all LD pups $(n=1,135$ cells, 10 nuclei, 5 mice).

In contrast, Perl:GFP circadian rhythms in SCN from LL pups were severely disrupted (Fig. $1 E$ ). The weak oscillations we observed in SCN from LL pups exhibited trough to peak amplitudes of 1.38 -fold on average (i.e., peaks $38 \%$ greater than troughs, $\pm 0.15,5$ mice). Group data demonstrated a transient increase in Perl:GFP during the first $12 \mathrm{~h}$ in vitro, perhaps due to the transition to in vitro conditions, but no evidence of sustained molecular rhythms in the population of SCN from LL pups, whether the data were aligned by local clock time (Fig. $1 F$ ), or by the time of peak expression in the first $24 \mathrm{~h}$ in vitro (not shown). In contrast to the whole SCN data, imaging of individual cellular rhythms within SCN from LL pups revealed the persistence of robust neuronal circadian rhythms in Perl promoter activation which were 1.5- to 4-fold in amplitude (Fig. 1G). The SCN neuronal rhythms of LL pups were, however, desynchronized in phase (Fig. 1G) and the peak times of individual neuronal rhythms from LL pups were widely dispersed (Fig. 1H). The SD of cell peak times for 
SCN nuclei from LL pups was significantly greater than that for cells in SCN of LD pups $(6.3 \pm 0.8 \mathrm{~h}, n=10$ nuclei, $p<0.01)$. Similar robust, but desynchronized, neuronal rhythms were observed in the SCN of all LL pups assayed ( $n=1,113$ cells, 10 nuclei, 5 mice). The differences in neuronal synchrony in SCN from LD and LL pups are illustrated in time-lapse videos (see supplementary Videos 1 and 2 at: http://www.cas.vanderbilt.edu/ PediatrRes2005OhtaMitchellMcMahon).

Dams in both LD and LL conditions during their lactation periods exhibited circadian behaviors with LD dams exhibiting rhythms with periods near $24 \mathrm{~h}(24.0 \pm 0.0 \mathrm{~h}, 5$ mice, Fig. $2 A, B)$ and LL dams showing slightly longer circadian periods $(25.1 \pm 0.8$ h, 5 mice, Fig. $2 C, D ; p<0.05) . \chi^{2}$ periodogram analysis revealed all dams had significant circadian periods $(p<0.05)$ and that there were no significant differences in the amplitude of locomotor rhythms in LL and LD dams $(p>0.05)$.

Effects of constant light on activity rhythms at later developmental stages. To gain insight into potential long-term effects of exposing the developing biological clock to constant light, P20 LL pups were subsequently exposed to LL for four weeks while their locomotor activity rhythms were monitored by wheel running. Interestingly, 67\% (26/39) of the LL-reared pups subsequently exposed to LL developed disrupted circadian locomotor activity patterns such as arhythmicity or multiple non-circadian rhythmic components (i.e., "splitting"; Fig. $3 A-C$ ) within the first seven weeks of life. In contrast, in a separate study in which P20 LD-reared pups were subsequently exposed to LL, all pups exhibited coherent circadian rhythms for several weeks and only after 3-5 mo in LL did approximately $10 \%$ of these mice finally develop disrupted circadian locomotor activity patterns (17). The LD cycle is a strong synchronizer known to reset the biological clock (1). To rescue the disrupted circadian behavioral phenotypes observed in LL-reared pups, LD cycles were applied to LL pups after P20. All these pups exhibited strong, coherent circadian locomotor rhythms synchronized to the LD cycle from their first day in LD (Fig. 3D-F).
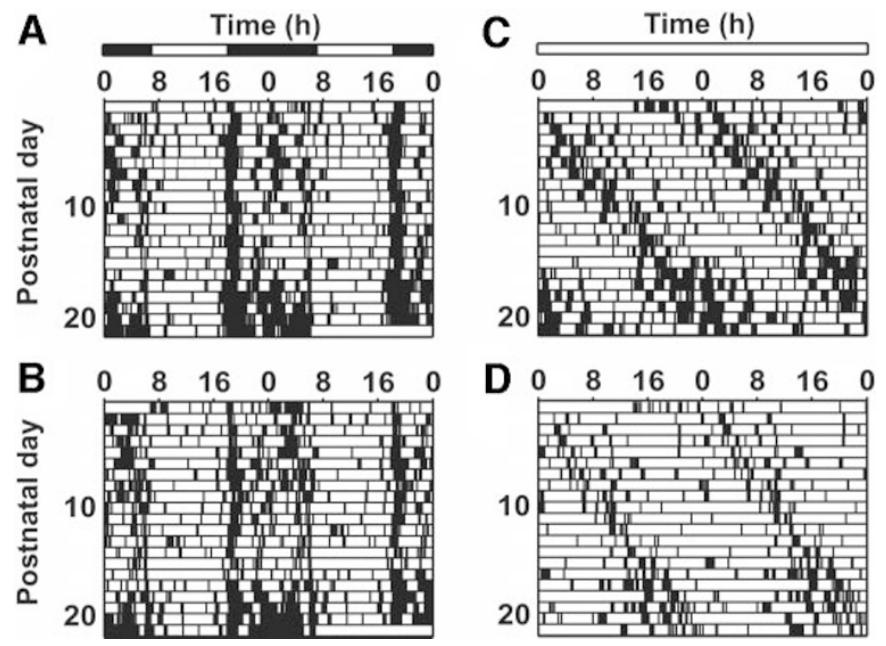

Figure 2. Behavioral rhythms from dams during their lactation periods. $(A, B)$ Actograms of wheel running activity from dam in LD. $(C, D)$ Actograms of wheel running activity from dams in LL. The open and solid bars on the top of the actograms represent light and dark periods, respectively.
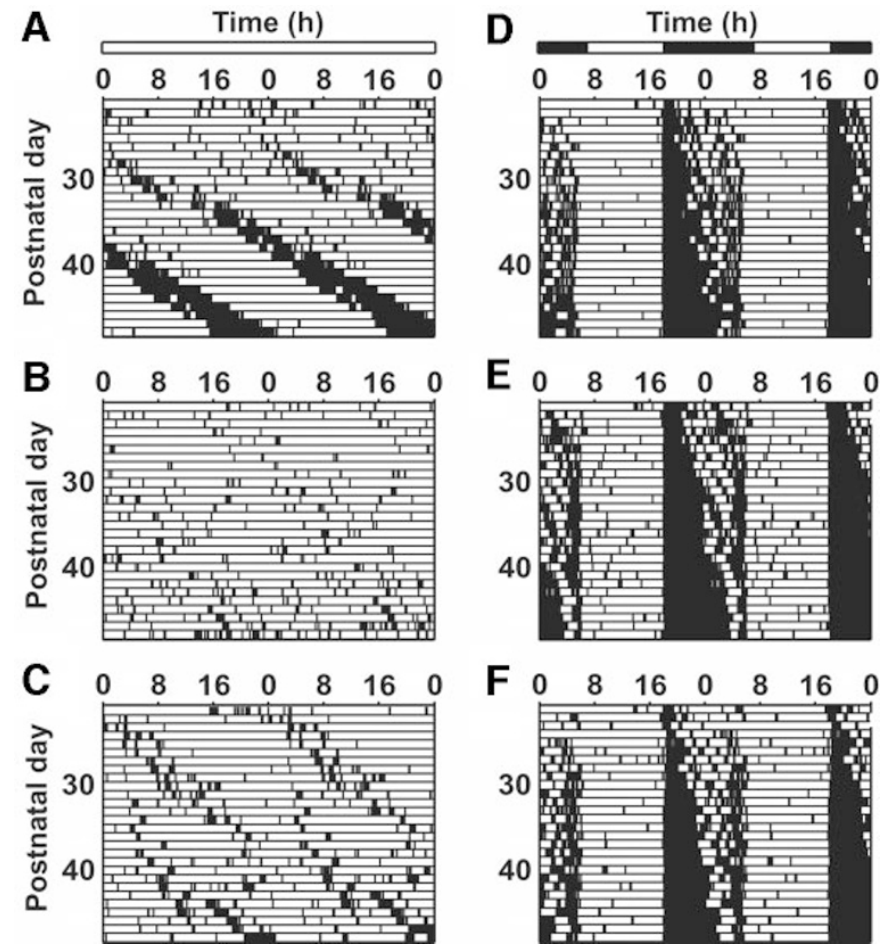

Figure 3. Behavioral rhythms from young mice raised continuously in constant light after birth $(A-C)$ and young mice raised in constant light for the first three weeks after birth and then transferred to LD on postnatal day 20 $(D-F)$. (A) Actogram of wheel running activity with circadian rhythmicity from an LL-treated pup. $(B, C)$ Actograms of wheel running activity with disrupted circadian rhythmicity from LL-treated pups. $(D-F)$ Actograms showing circadian activity of LD-treated pups.

\section{DISCUSSION}

Sensitivity of developing biological clocks to constant light. Our results provide compelling evidence that constant light disrupts developing biological clocks and overall circadian organization by desynchronizing the circadian rhythms of individual clock neurons within the SCN, but not by compromising the ability of single neurons to generate circadian rhythms. Cellular desynchronization is also the means by which constant light disrupts adult circadian clocks (17), but our data emphasize that developing biological clocks are more vulnerable to disruption by constant light and that cellular desynchronization during clock maturation can have longlasting effects. Finally, our results show that light-dark cycles can protect developing circadian clocks from disruption by irregular lighting conditions.

The disruption of cellular organization of the neonatal SCN by LL was reflected in disrupted circadian behaviors in weanling mice, demonstrating that lighting conditions have significant influence on neonatal biological clocks and their ability to maintain internal temporal order. Continuous 7-wk exposure to LL from birth induced $67 \%$ of developing mice to have disrupted circadian locomotor activities, whereas all mice which had been exposed to LD cycles for the first three weeks of life maintained coherent circadian behaviors for at least 3 mo in LL and only $10 \%$ subsequently developed circadian disruptions with 3-5 mo of LL exposure (17). This indicates a critical and lasting effect of constant light versus cyclic light 
on developing clocks for the first three weeks of life in mice. Whereas there are many previous studies regarding maternalfetal circadian interactions and its mechanisms (20-23) our studies concentrated on neonatal circadian development, holding lighting conditions during gestation constant, and then varying them during the neonatal period. Maternal-neonatal entrainment, in which pups synchronize to their mothers' circadian rhythms, has also been documented, but its physiologic mechanisms remain to be elucidated (24-27). Interestingly, our data demonstrates that even in the presence of maternal rhythmicity, light input can overcome maternal influence on pups' circadian rhythms, strongly influencing and even disrupting the maturing biological clock. Recently, vasoactive intestinal polypeptide (VIP) and gap junctions have been suggested to be key cellular coupling factors maintaining cellular synchrony within the SCN (28-30), and LL has been shown to suppress SCN VIP content in adult rodents (31). Developmental studies assessing the effects of LL on these modes of SCN neural communication may reveal the underlying mechanisms by which LL disrupts SCN maturation.

The neonatal period of SCN maturation in rodents is marked by the appearance of molecular clock gene rhythms as well as initiation of light responsiveness. In mice, the circadian rhythm of Perl mRNA expression starts at embryonic day 17 (E17), 2-4 d before birth (32), whereas in the rat, data indicate that Perl mRNA rhythms also begin between E20 and postnatal day 3 (P3) (33-36). The response of the SCN to light-dark cycles is already present at birth, as measured using c-fos gene induction (37-39). Recent studies have confirmed that the light entrainment pathway, consisting of intrinsically photoreceptive melanopsin-expressing retinal ganglion cells, is present and functional at P0 in both mice and rats $(40,41)$. Whereas the molecular development of the human biological clock and melanopsin systems is less well defined, human infants do show both a developmental time course in their circadian organization and evidence of circadian responses to light, suggesting the possibility of similar underlying mechanisms $(42,43)$.

Light-dark cycles as an effective chronotherapy for disrupted developing biological clocks. Our results show that constant light disrupts immature biological clocks and leads to abnormal circadian behaviors, however, once disrupted, coherent circadian behaviors could be restored by exposure to light-dark cycles. Exposure of the developing human circadian system to constant light also results in irregular sleep-wake patterns (7), reduced weight gain $(7,9)$ and various neurologic complications (44). These types of constant-light environments might be experienced in hospital intensive care units or in lighting conditions at home in our night-active modern society. Our data suggest that in these situations restoration of appropriate light-dark cycles can be a strong and simple chronotherapy to protect developing circadian clocks from the effects of irregular lighting conditions.

\section{REFERENCES}

1. Hastings MH, Reddy AB, Maywood ES 2003 A clockwork web: circadian timing in brain and periphery, in health and disease. Nat Rev Neurosci 4:649-661
2. Pittendrigh CS 1960 Circadian rhythms and the circadian organization in living systems. Cold Spring Harb Symp Quant Biol 25:159-184

3. Aschoff J, Wever R 1976 Human circadian rhythms: a multioscillatory system. Fed Proc 35:2326-2332

4. Daan S, Pittendrigh CS 1976 A functional analysis of circadian pacemakers in nocturnal rodents. III. Heavy water and constant light: homeostasis of frequency? J Comp Physiol A Neuroethol Sens Neural Behav Physiol 106:267-290

5. Benson JA, Jacklet JW 1977 Circadian rhythm of output from neurones in the eye of aplysia: 3. Effects of light on clock and receptor output measured in the optic nerve. J Exp Biol 70:183-194

6. Depres-Brummer P, Levi F, Metzger G, Touitou Y 1995 Light-induced suppression of the rat circadian system. Am J Physiol 268:R1111-R1116

7. Mann NP, Haddow R, Stokes L, Goodley S, Rutter N 1986 Effect of night and day on preterm infants in a newborn nursery: randomised trial. BMJ (Clin Res Ed) 293:1265-1267

8. Mirmiran M, Ariagno RL 2000 Influence of light in the NICU on the development of circadian rhythms in preterm infants. Semin Perinatol 24:247-257

9. Miller CL, White R, Whitman TL, O'Callaghan MF, Maxwell SE 1995 The effects of cycled versus noncycled lighting on growth and development in preterm infants. Infant Behav Dev 18:87-95

10. Brandon DH, Holditch-Davis D, Belyea M 2002 Preterm infants born at less than 31 weeks' gestation have improved growth in cycled light compared with continuous near darkness. J Pediatr 140:192-199

11. Fielder AR, Moseley MJ 2000 Environmental light and the preterm infant. Semin Perinatol 24:291-298

12. Kennedy KA, Fielder AR, Hardy RJ, Tung B, Gordon DC, Reynolds JD LIGHTROP Cooperative Group 2001 Reduced lighting does not improve medical outcomes in very low birth weight infants. J Pediatr 139:527-531.

13. [no listed authors] 2003 The creation of formal planning guidelines for newborn intensive care units (NICUs). J Perinatol 23:S4-S21.

14. Kuhlman SJ, Quintero JE, McMahon DG 2000 GFP fluorescence reports Period 1 circadian gene regulation in the mammalian biological clock. Neuroreport 11:14791482

15. LeSauter J, Yan L, Vishnubhotla B, Quintero JE, Kuhlman SJ, McMahon DG, Silver R 2003 A short half-life GFP mouse model for analysis of suprachiasmatic nucleus organization. Brain Res 964:279-287

16. Quintero JE, Kuhlman SJ, McMahon DG 2003 The biological clock nucleus: a multiphasic oscillator network regulated by light. J Neurosci 23:8070-8076

17. Ohta H, Yamazaki S, McMahon DG 2005 Constant light desynchronizes mammalian clock neurons. Nat Neurosci 8:267-269

18. Sokolove PG, Bushell WN 1978 The chi square periodogram: its utility for analysis of circadian rhythms. J Theor Biol 72:131-160

19. Canal-Corretger MM, Vilaplana J, Cambras T, Diez-Noguera A 2001 Effect of light on the development of the circadian rhythm of motor activity in the mouse. Chronobiol Int 18:683-696

20. Reppert SM, Schwartz WJ 1983 Maternal coordination of the fetal biological clock in utero. Science 220:969-971

21. Reppert SM, Weaver D, Rivkees S 1988 Maternal communication of circadian phase to the developing mammal. Psychoneuroendocrinology 13:63-78

22. Weaver DR, Reppert SM 1989 Periodic feeding of SCN-lesioned pregnant rats entrains the fetal biological clock. Brain Res Dev Brain Res 46:291-296

23. Dolatshad H, Campbell EA, O'Hara L, Maywood ES, Hastings MH, Johnson MH 2006 Developmental and reproductive performance in circadian mutant mice. Hum Reprod 21:68-79

24. Hiroshige T, Honma K, Watanabe K 1982 Possible zeitgebers for external entrainment of the circadian rhythm of plasma corticosterone in blind infantile rats. J Physiol 325:507-519

25. Takahashi K, Deguchi T 1983 Entrainment of the circadian rhythms of blinded infant rats by nursing mothers. Physiol Behav 31:373-378

26. Reppert SM, Coleman RJ, Heath HW, Swedlow JR 1984 Pineal N-acetyltransferase activity in 10-day-old rats: a paradigm for studying the developing circadian system. Endocrinology 115:918-925

27. Duffield GE, Ebling FJ 1998 Maternal entrainment of the developing circadian system in the Siberian hamster (Phodopus sungorus). J Biol Rhythms 13:315-329

28. Aton SJ, Colwell CS, Harmar AJ, Waschek J, Herzog ED 2005 Vasoactive intestinal polypeptide mediates circadian rhythmicity and synchrony in mammalian clock neurons. Nat Neurosci 8:476-483

29. Maywood ES, Reddy AB, Wong GK, O'Neill JS, O'Brien JA, McMahon DG, Harmar AJ, Okamura H, Hastings MH 2006 Synchronization and maintenance of timekeeping in suprachiasmatic circadian clock cells by neuropeptidergic signaling. Curr Biol 16:599-605

30. Long MA, Jutras MJ, Connors BW, Burwell RD 2005 Electrical synapses coordinate activity in the suprachiasmatic nucleus. Nat Neurosci 8:61-66

31. Isobe Y, Nishino H 1998 AVP rhythm in the suprachiasmatic nucleus in relation to locomotor activity under constant light. Peptides 19:827-832

32. Shimomura H, Moriya T, Sudo M, Wakamatsu H, Akiyama M, Miyake Y, Shibata S 2001 Differential daily expression of Per1 and Per2 mRNA in the suprachiasmatic nucleus of fetal and early postnatal mice. Eur J Neurosci 13:687-693

33. Ohta H, Honma S, Abe H, Honma K 2002 Effects of nursing mothers on rPer1 and rPer2 circadian expressions in the neonatal rat suprachiasmatic nuclei vary with developmental stage. Eur J Neurosci 15:1953-1960

34. Ohta H, Honma S, Abe H, Honma K 2003 Periodic absence of nursing mothers 
phase-shifts circadian rhythms of clock genes in the suprachiasmatic nucleus of rat pups. Eur J Neurosci 17:1628-1634

35. Sladek M, Sumova A, Kovacikova Z, Bendova Z, Laurinova K, Illnerova H 2004 Insight into molecular core clock mechanism of embryonic and early postnatal rat suprachiasmatic nucleus. Proc Natl Acad Sci USA 101:6231-6236

36. Kovacikova Z, Sladek M, Laurinova K, Bendova Z, Illnerova H, Sumova A 2005 Ontogenesis of photoperiodic entrainment of the molecular core clockwork in the rat suprachiasmatic nucleus. Brain Res 1064:83-89

37. Leard LE, Macdonald ES, Heller HC, Kilduff TS 1994 Ontogeny of photic-induced c-fos mRNA expression in rat suprachiasmatic nuclei. Neuroreport 5:2683-2687

38. Weaver DR, Reppert SM 1995 Definition of the developmental transition from dopaminergic to photic regulation of c-fos gene expression in the rat suprachiasmatic nucleus. Brain Res Mol Brain Res 33:136-148

39. Munoz Llamosas M, Huerta JJ, Cernuda-Cernuda R, Garcia-Fernandez JM 2000
Ontogeny of a photic response in the retina and suprachiasmatic nucleus in the mouse. Brain Res Dev Brain Res 120:1-6

40. Hannibal J, Fahrenkrug J 2004 Melanopsin containing retinal ganglion cells are light responsive from birth. Neuroreport 15:2317-2320

41. Sekaran S, Lupi D, Jones SL, Sheely CJ, Hattar S, Yau KW, Lucas RJ, Foster RG, Hankins MW 2005 Melanopsin-dependent photoreception provides earliest light detection in the mammalian retina. Curr Biol 15:1099-1107

42. Hao H, Rivkees SA 1999 The biological clock of very premature primate infants is responsive to light. Proc Natl Acad Sci USA 96:2426-2429

43. Rivkees SA, Mayes L, Jacobs H, Gross I 2004 Rest-activity patterns of premature infants are regulated by cycled lighting. Pediatrics 113:833-839

44. Als H, Lawhon G, Duffy FH, McAnulty GB, Gibes-Grossman R, Blickman JG 1994 Individualized developmental care for the very low-birth-weight preterm infant. Medical and neurofunctional effects. JAMA 272:853-858 